\title{
Ultra-Wideband Transmitted-Reference Impulse Radio System With Multiple Pulse Types
}

\author{
$\mathrm{Xu} \mathrm{Lu}$, and Wen Chen \\ Department of Electronic Engineering \\ Shanghai Jiao Tong University \\ Shanghai, China 200240 \\ Email: \{jarodlx, wenchen\}@sjtu.edu.cn
}

\begin{abstract}
In this paper, we analyze the performance of transmitted-reference (TR) impulse radio (IR) system with multiple pulse types, using the autocorrelation receiver (AcR) instead of Rake receiver. We obtain the exact expression of symbol error probability (SEP) without considering of the inter-frame interference (IFI) and multiple access interference (MAI). Then we discuss the capability of multi-pulse TR-IR system against the IFI. By observing the result of simulation, we find that the multi-pulse TR-IR system can achieve better BEP performance than the conventional single pulse system in the IFI environment.
\end{abstract}

Index Terms-multiple pulse types, autocorrelation receiver (AcR), transmitted-reference (TR).

\section{INTRODUCTION}

Since the Federal Communications Commission (FCC) allocated the bandwidth from $3.1 \mathrm{GHz}$ to $10.6 \mathrm{GHz}$ to ultra-wide band (UWB) communication in 2002, the UWB technology has attracted great attentions in the world due to its great potentials of variety applications such as short-range highspeed wireless transmission and precise location. Commonly, impulse radio (IR) systems, which transmit a train of extremely short pulses, are employed to implement UWB systems [1], [2]. In an IR system, each symbol is transmitted in a number $N_{f}$ of pulses, and usually there are two cases to carry the users information: stored-reference (SR) system and transmittedreference (TR) system. Stored-reference (SR) system is introduced in [3]. In our paper we focus on the transmittedreference (TR) system.

The study of the transmitted-reference (TR) system was started more than 50 years ago [4], [5]. The transmission of a reference and data signal pair was separated either in time [5], or in frequency [6]. In other words, out of the $N_{f}$ pulses transmitted per information symbol, half of them are used as reference pulses, whereas the rest half are used as data pulses. Like the differential systems, the information is carried by the relative polarity of the reference and the data pulses. So there is no need to estimate the channel because the reference and the data pulses experience the same channel, assuming the time spacing be less than the coherence time. In this case we can simply use an autocorrelation receiver (AcR) instead of Rake receiver.

In the conventional impulse radio (IR) system, a single type of UWB pulse is transmitted in all frames of all users [1].
While recently in [7], an impulse radio system with multiple pulse types was proposed and the performance analysis of stored-reference (SR) system was given in [8]. The advantages of multiple pulse types over the conventionally single pulse case are attractive. By using the multiple pulse types, the multiple access interference (MAI) and inter-frame interference (IFI) can be dramatically suppressed [8]. But the author only gave the performance analysis of SR system. So in our paper, we analyze the performance of the transmitted-reference impulse radio (TR-IR) system employing different types of orthogonal pulses. As the conventional receiver of transmittedreference signaling [9], the autocorrelation receiver (AcR) is employed.

The rest of the paper is organized as follows. In Section II, we introduce the model of the transmitted-reference impulse radio (TR-IR) system with multiple pulse types. In Section III, we analyze the symbol error probability (SEP) of the system, by neglecting the multiple access interference (MAI) and inter-frame interference (IFI). In Section IV, we discuss a more complicated situation with the existence of IFI. Finally, in Section V, we conclude the paper.

\section{MULTI-PULSE TR-IR SYSTEM MODEL}

The transmitted signal from the $k$-th user in a multi-pulse TR-IR system can be generally expressed as [8]

$$
s^{(k)}(t)=\frac{1}{\sqrt{N_{f}}} \sum_{i=-\infty}^{\infty} \sum_{n=0}^{N_{p}-1} s_{i, n}^{(k)}(t)
$$

where $N_{f}$ is the number of pulses transmitted per information symbol, $N_{p}$ is the number of different pulse types, and $s_{i, n}^{(k)}(t)$ which represents the UWB pulses of type $n$ transmitted for the $i$-th information symbol of user $k$ is given as

$$
s_{i, n}^{(k)}(t)=\sum_{j=i N_{f} /\left(2 N_{p}\right)}^{(i+1)\left(N_{f} / 2 N_{p}\right)-1}\left\{g_{n}^{(k)}(t)+b_{\left\lfloor 2 N_{p} j / N_{f}\right\rfloor}^{(k)} g_{n}^{(k)}\left(t-T_{n}^{(k)}\right)\right\}
$$

where $b_{\left\lfloor 2 N_{p} j / N_{f}\right\rfloor}^{(k)}$ carries the information of the user. Note that we assume that the number of pulses per symbol $N_{f}$ to be an even multiple of $N_{p}$ for simplicity of notation. And $T_{n}^{(k)}$ represents the interval between the two pulses in a pair of type 
$n$ for user $k \cdot g_{n}^{(k)}(t)$ is given as

$$
g_{n}^{(k)}(t)=d_{2 j N_{p}+n}^{(k)} p_{n}^{(k)}\left(t-\left(2 j N_{p}+n\right) T_{f}-c_{2 j N_{p}+n}^{(k)} T_{c}\right)
$$

where $d_{j}^{(k)}$ represents the polarity code, which eliminates the discrete line of power spectrum density (PSD) [10] and provides robustness against multiple access interference (MAI) [11]. The time-hopping (TH) code for user $k$ is denoted by $c_{j}^{(k)}$, taking the values in the set $\left\{0,1, \ldots, N_{c}-1\right\}$. Here $N_{c}$ is the number of chips per frame. $p_{n}^{(k)}(t)$ is the $n$-th type pulse of the $k$-th user, and all types of the pulses have the same pulse energy $E_{p}$. For the convenience of notation, the pulses are normalized so that $\int_{0}^{T_{c}}\left|p_{n}^{(k)}(t)\right|^{2}=1 . T_{f}$ and $T_{c}$ represent the frame interval and chip interval respectively.

The channel is modeled as

$$
h(t)=\sum_{l=1}^{L} \alpha_{l} \delta\left(t-\tau_{l}\right)
$$

where $\alpha_{l}$ and $\tau_{l}$ denote the attenuation and delay of $l$-th path respectively, and $L$ is the number of multipath components. So the received signal can be written as

$$
r(t)=\int_{-\infty}^{+\infty} h(\tau) s(t-\tau) d \tau+n(t)
$$

where $n(t)$ is zero-mean, white Gaussian noise with two-sided power spectral density $N_{0} / 2$. From here on, we drop the user index $k$ for notational convenience.

\section{SEP ANALYSIS OF ACR FOR MULTI-PULSE TR-IR SYSTEM}

In this section, we assume the channel is slow-fading and there is no inter-frame interference (IFI) existing. While in section IV, we will discuss the situation with IFI.

In TR signaling, the autocorrelation receiver (AcR) is widely employed because it is simple-implemented and can exploit the diversity inherent in the multipath channel [12]. So we can still use the AcR in the multi-pulse types situation after some modification. First we rewrite the received signal

$$
r(t)=\sum_{n=1}^{N_{p}} h(t) * s_{n}(t)+n(t)=\sum_{n=1}^{N_{p}} r_{n}(t)
$$

where $*$ denotes the convolution calculation. Note that for simplicity of analysis, the noise $n(t)$ is already divided into $N_{p}$ noises which contained by each $r_{n}(t)$. The autocorrelation receiver is shown in Fig. 1, the received signal first passes a bandpass zonal filter (BPZF) with bandwidth $W$ and center frequency $f_{c}$ to eliminate the out-of-band noise. No distortion or overlap caused by filtering is considered. The switch is connected to every branch continuously for the interval of one frame $T_{f}$. So in each branch, the pulses are in the same type. Every branch consists of an autocorrelation receiver which generates $N_{f} / 2 N_{p}$ correlated values, and the delay $T_{n}$ in each branch are different from the others. By combining $N_{p}$ branches, we form a decision statistic $Z_{i}$ for detection of the transmitted data symbol. In the analysis of each branch, we use

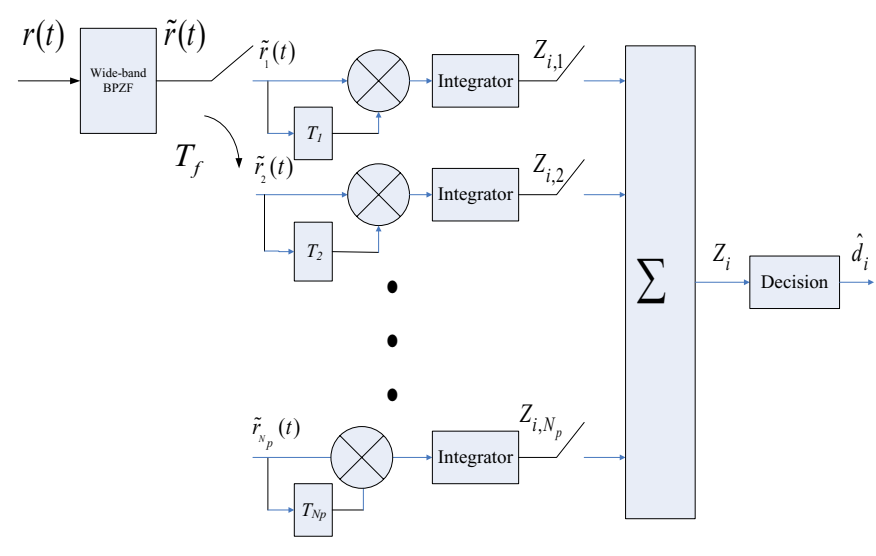

Fig. 1. AcR for Multi-pulse TR-IR signaling

the method proposed in [12]. Without loss of generality, we analyze the first branch for instance and the rest of branches share the same processing. The decision values generated by first branch can be expressed as

$$
Z_{i, 1}=\sum_{j=0}^{\left(N_{f} / 2 N_{p}\right)-1} \int_{j N_{p} T_{f}+T_{1}}^{j N_{p} T_{f}+T_{1}+T} \tilde{r}_{1}(t) \tilde{r}_{1}\left(t-T_{1}\right) d t
$$

where $\tilde{r}_{1}(t)$ and $\tilde{n}(t)$ are the received signal and noise at the output of the BPZF respectively. Using the (2) and (3), we can rewrite equation (7)

$Z_{i, 1}=\sum_{j=0}^{\left(N_{f} / 2 N_{p}\right)-1} \int_{0}^{T}\left(w_{j}(t)+\eta_{1, j}(t)\right)\left(d_{i} w_{j}(t)+\eta_{2, j}(t)\right) d t$

where $w_{j}(t) \triangleq \breve{g}_{1}\left(t+j 2 N_{p} T_{f}+i N_{f} T_{f}\right), \eta_{1, j}(t) \triangleq \tilde{n}(t+$ $\left.j 2 N_{p} T_{f}+i N_{f} T_{f}\right)$ and $\eta_{2, j}(t) \triangleq \tilde{n}\left(t+j 2 N_{p} T_{f}+i N_{f} T_{f}+T_{1}\right)$, by denoting $\breve{g}_{1}(t)=g_{1}(t) * h(t) * h_{Z F}(t)$, and $h_{Z F}(t)$ is the impulse response of the BPZF. After some manipulation, $Z_{i, 1}$ can be expressed as

$$
\begin{aligned}
Z_{i, 1}= & \sum_{\substack{j=0\\
}}^{\left(N_{f} / 2 N_{p}\right)-1} \int_{0}^{T}\left\{d_{i} w_{j}^{2}(t)+w_{j}(t) \eta_{2, j}(t)\right. \\
& \left.+d_{i}(t) \eta_{1, j}(t)+\eta_{1, j}(t) \eta_{2, j}(t)\right\} d t
\end{aligned}
$$

By applying the sampling theorem proposed in [5], $Z_{i, 1}$ can be rewritten as follows

$$
\begin{gathered}
Z_{i, 1}=\sum_{j=0}^{\left(N_{f} / 2 N_{p}\right)-1} U_{i, j} \\
U_{i, j}=\sum_{\substack{k=1 \\
+}}^{2 W T} d_{i} w_{j, k}^{2}+w_{j, k} \eta_{2, j}, k \\
\end{gathered}
$$

where $1 / W$ is the sampling interval, $w_{j, k}, \eta_{1, j, k}$ and $\eta_{2, j, k}$ are the $k$-th sample of $w_{j}(t), \eta_{1, j}(t)$ and $\eta_{2, j}(t)$ in (9) 


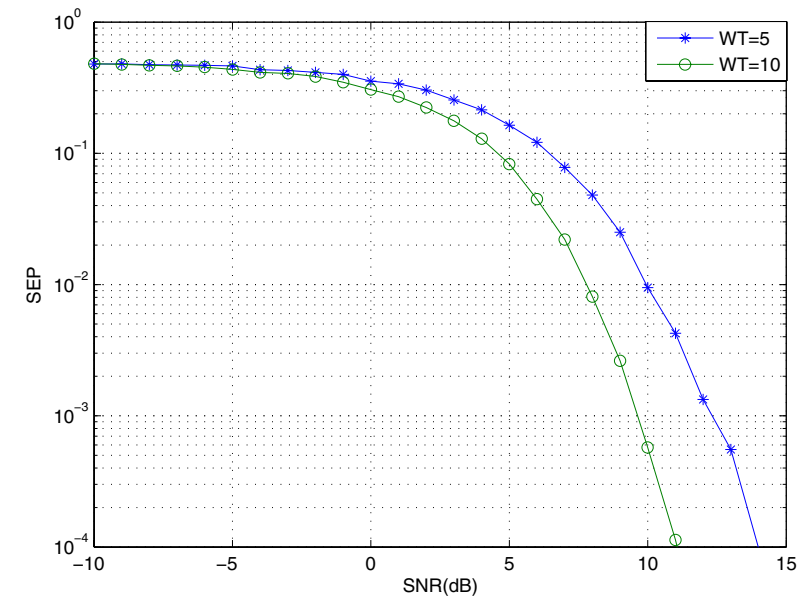

Fig. 2. The SEP of the AcR in i.i.d. Nakagami- $m$ fading channels with $m=2.0$

respectively. Conditioned on $d_{i},(11)$ can be expressed as

$$
\begin{array}{r}
U_{i, j \mid d_{i}=+1}=\sum_{k=1}^{2 W T}\left[\left(w_{j, k}+\beta_{1, j, k}\right)^{2}-\beta_{2, j, k}^{2}\right] \\
U_{i, j \mid d_{i}=-1}=\sum_{k=1}^{2 W T}\left[-\left(w_{j, k}-\beta_{2, j, k}\right)^{2}+\beta_{1, j, k}^{2}\right]
\end{array}
$$

where $\beta_{1, j, k}=\left(\eta_{2, j, k}+\eta_{1, j, k}\right) / 2$ and $\beta_{2, j, k}=\left(\eta_{2, j, k}-\right.$ $\left.\eta_{1, j, k}\right) / 2$. Note that $\beta_{1, j, k}$ and $\beta_{2, j, k}$ are independent Gaussian random variables. Then, we define $X_{1}$ and $Y_{1}$ as

$$
\begin{gathered}
X_{1}=\frac{1}{2 \sigma^{2}} \sum_{j=0}^{\left(N_{f} / 2 N_{p}\right)-1} \sum_{k=1}^{2 W T}\left(w_{j, k}+\beta_{1, j, k}\right)^{2} \\
Y_{1}=\frac{1}{2 \sigma^{2}} \sum_{j=0}^{\left(N_{f} / 2 N_{p}\right)-1} \sum_{k=1}^{2 W T} \beta_{2, j, k}^{2}
\end{gathered}
$$

where $\sigma^{2}$ is the variance of $\beta_{1, j, k}$. The probability density functions (pdfs) of $X_{1}$ and $Y_{1}$ conditioned on $\gamma$ are given by

$$
\begin{gathered}
f_{X_{1} \mid \gamma}\left(x_{1}\right)=I_{q-1}\left(2 \sqrt{2 x_{1} \gamma}\right) \exp \left\{-\left(x_{1}+2 \gamma\right)\right\}\left(\frac{x_{1}}{2 \gamma}\right)^{(q-1) / 2} \\
f_{Y_{1} \mid \gamma}\left(y_{1}\right)=\frac{y_{1}^{q-1}}{(q-1) !} \exp \left\{-y_{1}\right\}
\end{gathered}
$$

where $I_{q-1}(\cdot)$ denotes the $(q-1)$-th order Bessel function of the first kind, and $\gamma$ which represents the output SNR per symbol, is defined as $\gamma=\frac{N_{f} E_{p}}{2 N_{0} N_{p}} \sum_{l=1}^{W T} \alpha_{l}^{2}$. Note that $f_{X_{1} \mid \gamma}\left(x_{1}\right)$ and $f_{Y_{1} \mid \gamma}\left(y_{1}\right)$ are the pdfs of the noncentral and central chi-squared random variables with $2 q$ degrees of freedom respectively with $q=\frac{N_{f} W T}{2 N_{p}}$. In fact, such analysis of conventional TR-IR AcR was given in [12].

Now, we can form the decision statistic by combing all the $N_{p}$ branches together, $Z_{i}=\sum_{n=1}^{N_{p}} Z_{i, n}$. So we can define $X$ and $Y$ as

$$
X=\sum_{n=1}^{N_{p}} X_{n}, Y=\sum_{n=1}^{N_{p}} Y_{n}
$$

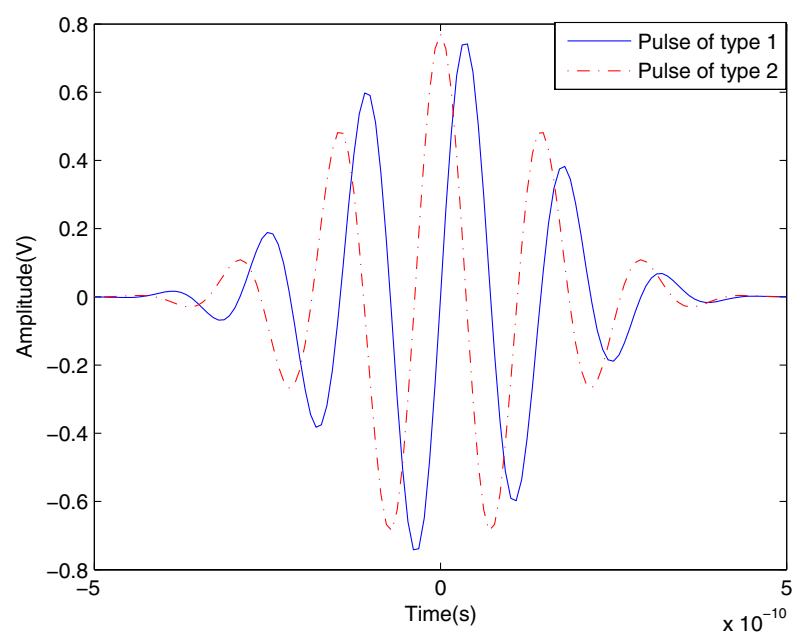

Fig. 3. The orthogonal pulses generated by the eigenvalue decomposition algorithm

Because $X_{1}, X_{2}, \ldots, X_{N_{p}}$ are independent chi-squared random variables, so $X$ is a chi-squared random variable with $2 N_{p} q$ degrees of freedom. Obviously, $Y$ is also a chi-squared random variable with $2 N_{p} q$ degrees of freedom.

We find that the expression of SEP of multi-pulse system is the same as the conventional system [12]. And it is easy to understand this result according to the neglecting of MAI and IFI. So the SEP of AcR of the multi-pulse TR-IR system can be expressed as

$P_{e}=\frac{1}{2^{q}} \sum_{i=0}^{q-1}\left(\frac{E\left[(\gamma)^{2} \exp (-\gamma)\right]}{i !} \sum_{j=i}^{q-1} \frac{1}{2^{j}} \frac{(j+q-1) !}{(j-i) !(q+i-1) !}\right)$

Note that here $q=\frac{N_{f} W T}{2}$ and $\gamma=\frac{N_{f} E_{p}}{2 N_{0}} \sum_{l=1}^{W T} \alpha_{l}^{2}$.

Fig. 2 shows the SEP performance of the AcR. Here we set the channel model as Nakagami- $m$ fading channel with $L=40, m=2.0$. The number of frames per symbol $N_{f}=16$, and $W T$ is chosen as 5,10 respectively.

\section{MULTI-PULSE TR-IR SYSTEM WITH IFI}

In this section, we extend our discussion to a more complicated situation with considering of the inter-frame interference (IFI). In this case, the transmitted reference signal used as a correlator template is noisy and interfered, so it is very difficult to calculate the pdf of the inter-frame interference (IFI) and the exact expression of BEP. But it is easy to accept the fact that multi-pulse type TR system should have better capability against IFI compared with single pulse type system, because the transmitted pulses are orthogonal between every two adjacent frames. And the result of simulation, in which we compare the BEP performance between single pulse type and double pulse types proves this point. The orthogonal pulses are generated by the algorithm proposed in [13]. The pulses and their cross-correlation function are shown in Fig. 3 and Fig. 4 respectively. 


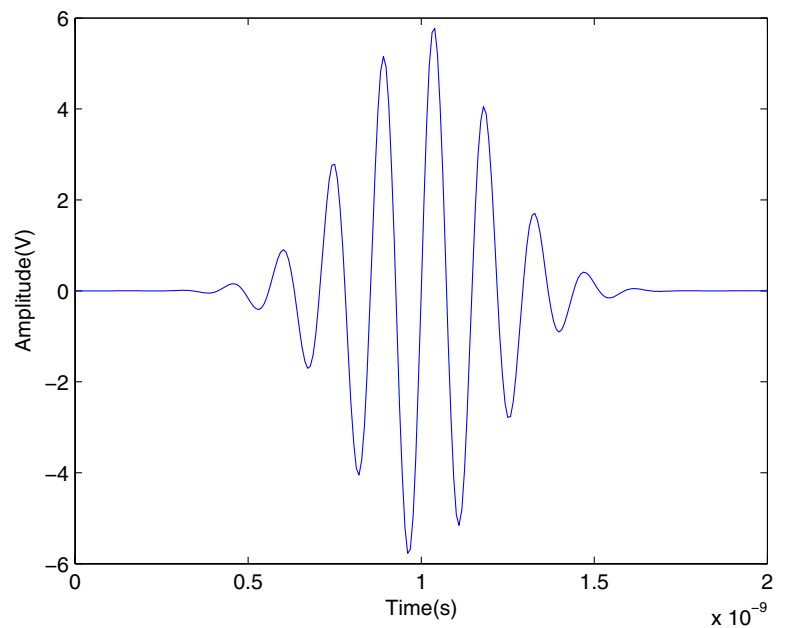

Fig. 4. The cross-correlation function of the transmitted pulse types

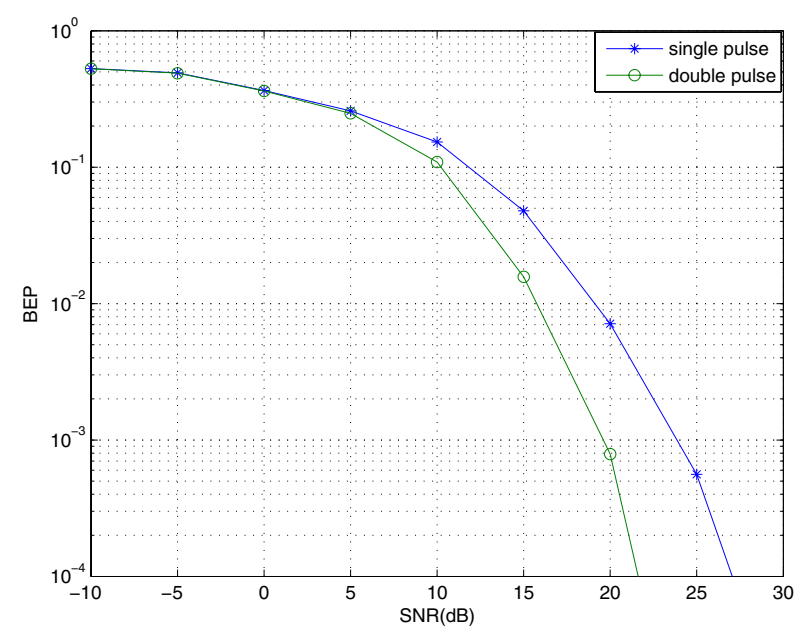

Fig. 5. BEP performance of the single pulse and double pulse TR-IR systems using AcR

Fig. 5 shows the BEP performance of AcR for single pulse type and double pulse types. All the channels are restricted to i.i.d. Nakagami- $m$ fading channels with $L=40, m=2.0$. We can observe the effects of double pulse types on reducing IFI compared with single pulse type.

\section{CONCLUSION}

In this paper, we analyzed the performance of a TR-IR system using autocorrelation receiver. When neglecting the inter-frame interference (IFI) and multiple access interference (MAI), we obtained the exact expression of SEP, which is the same as the conventional TR-IR system employing single pulse type. While in the IFI environment, we found the performance of multi-pulse type system is better than the conventional system after the BEP simulation. If without considering of the complexity of receivers, more improvements can be achieved by increasing the number of the pulse types.

\section{ACKNOWLEDGMENT}

This work is supported by NSF China \#60672067, by NSF Shanghai \#06ZR14041, by Shanghai-Canada NRC \#06SN07112, by Cultivation Fund of the Key Scientific and Technical Innovation Project, Ministry of Education of China \#706022, by Program for New Century Excellent Talents in University \#NCET-06-0386, and by PUJIANG Talents \#07PJ4046.

\section{REFERENCES}

[1] M. Z. Win and R. A. Scholtz, "Impulse radio: How it works," IEEE Commun. Lett., vol. 2, pp. 36-38, Feb. 1998.

[2] R. Hoctor and H. Tomlinson, "Delay-hopped transmitted-reference RF communications," in Proc. IEEE Conf. Ultra Wide-band Syst. Technol., Baltimore, MD, May 2002, pp. 265-269.

[3] M. H. Chung and R. A. Scholtz, "Comparison of transmitted- and storedreference systems for ultra-wideband communications," in Proc. IEEE Military Commun. Conf., Monterey, CA, Oct. 31-Nov. 32004.

[4] C. Rushforth, "Transmitted-reference techniques for random or unknown channels," IEEE Trans. Inform. Theory, vol. 10, pp. 39-42, Jan. 1964.

[5] R. Gagliardi, "A geometrical study of transmitted reference communication system," IEEE Trans. Commun., pp. 118-123 Dec. 1964.

[6] A. Polydoros and K. T. Woo, "LPI detection of frequency-hopping signals using autocorrelation techniques," IEEE J. Select. Areas Commun., vol. 3, no. 5, pp. 714-726, Sept. 1985.

[7] H. Harada, K. Ikemoto, and R. Kohno, "Modulation and hopping using modified hermite pulses for UWB communications," in Proc. IEEE Conf. Ultra Wide-band Syst.Technol., Kyoto, Japan, May 2004, pp. 336-340.

[8] S. Gezici, Z. Sahinoglu, H. Kobayashi, and H. V. Poor, "Ultra-Wideband impulse radio systems with multiple pulse types," IEEE J. Select. Areas Commun., vol. 24, no. 4, pp. 892-898, Apr. 2006.

[9] Y. L. Chao and R. A. Scholtz, "Ultra-wide transmitted reference systems," IEEE Trans. Veh. Technol. Invited paper, vol. 54, no.5, pp. 1556-1568, Sep. 2005.

[10] Y. P. Nakache and A. F. Molisch, "Spectral shape of UWB signals for time-hopping impulse radio," IEEE J. Select. Areas Commun., vol. 24, no.4, pp. 738-744, Apr. 2006.

[11] E. Fishler and H. V. Poor, "On the tradeoff between two types of processing gain," IEEE Trans. Veh. Technol. Conf., vol. 4, Jeju, Korea, April 2003, pp. 2510-2514.

[12] T. Q. Quek and M. Z. Win, "Ultrawide bandwidth transmitted-reference signaling," in Proc. IEEE ICC., vol. 6, Paris, France, Jun. 2004, pp. 34093413.

[13] Brent Parr, ByungLok Cho, et al, "A novel ultra-wideband pulse design algorithm,” IEEE Commun. Lett., vol. 54, no.5, pp. 1556-1568, Sep. 2005. 